\title{
ENTROPY BUMPS AND ANOTHER SUFFICIENT CONDITION FOR THE TWO-WEIGHT BOUNDEDNESS OF SPARSE OPERATORS
}

\author{
ROBERT RAHM AND SCOTT SPENCER
}

\begin{abstract}
In this short note, we give a very efficient proof of a recent result of TreilVolberg and Lacey-Spencer giving sufficient conditions for the two-weight boundedness of a sparse operator. We also give a new sufficient condition for the two-weight boundedness of a sparse operator. We make critical use of a formula of Hytönen in [6].
\end{abstract}

\section{INTRODUCTION}

Let $\mathcal{D}$ be a dyadic lattice. Recall that a collection $\mathcal{S}$ of cubes in $\mathcal{D}$ is said to be sparse if the following holds, uniformly over $\mathrm{P} \in \mathcal{D}:\left|\cup_{\mathrm{Q} \in \mathcal{S}: S \subset \mathrm{P}} \mathrm{Q}\right| \leq \frac{1}{2}|\mathrm{P}|$. This implies that the following holds, uniformly over all $\mathrm{P} \in \mathcal{D}: \sum_{\mathrm{Q} \in \mathcal{S}: S \subset \mathrm{P}}|\mathrm{Q}| \lesssim|\mathrm{P}|$. For a cube $\mathrm{Q} \in \mathcal{S}$, let $\mathrm{E}_{\mathrm{Q}}:=\mathrm{Q} \backslash \cup_{S \in \mathcal{S}: S \subset \mathrm{Q}} S$ and note that $\left|\mathrm{E}_{\mathrm{Q}}\right| \simeq|\mathrm{Q}|$. The sparse operator $\mathrm{T}_{\mathcal{S}}$ indexed over cubes in $\mathcal{S}$ is defined by $T_{\mathcal{S}} f(x):=\sum_{S \in \mathcal{S}}\langle f\rangle_{S} \mathbb{1}_{\mathcal{S}}(x)$. Here and below, $\langle f\rangle_{\mathrm{Q}}:=|\mathrm{Q}|^{-1} \int_{\mathrm{Q}} f(x) \mathrm{d} x$.

Due to deep and important theorems of Lerner, Lacey, and Rey and Conde-Alonso $[1,9,12]$ important operators in harmonic analysis (for example, maximal functions, Calderón-Zygmund Operators, Haar shifts) are point wise dominated by finite sums of sparse operators. Thus, proving two-weight inequalities for these sparse operators will imply the same theorems for other operators of interest.

Recently, a sufficient condition for the two-weight boundedness of sparse operators was provided by Treil-Volberg and Lacey-Spencer $[13,19]$. The conditions are in terms of so-called "entropy bumps" introduced in [19]. We give an efficient proof of these results and also give a new condition. The main results are as follows. Throughout, let

$$
\rho_{\sigma}(\mathrm{Q}):=\frac{1}{\sigma(\mathrm{Q})} \int_{\mathrm{Q}} \mathrm{M}\left(\mathbb{1}_{\mathrm{Q}} \sigma\right)(\mathrm{x}) \mathrm{d} x, \quad[w, \sigma]_{\mathrm{p}, \mathfrak{\varepsilon}_{\mathrm{p}}}:=\sup _{\mathrm{Q} \text { a cube }}\langle w\rangle_{\mathrm{Q}}\langle\sigma\rangle_{\mathrm{Q}}^{\mathrm{p}-1} \rho_{\sigma}(\mathrm{Q}) \mathcal{E}_{\mathrm{p}}\left(\rho_{\sigma}(\mathrm{Q})\right) .
$$

where $\varepsilon_{\mathfrak{p}}$ is an increasing function on $[1, \infty]$ satisfying $\sum_{r \in \mathbb{N}} \varepsilon_{\mathfrak{p}}\left(2^{r}\right)^{-\frac{1}{p}}<\infty$. Our first theorem is the following

Theorem 1.1. With definitions as above, and $1<p<\infty$, there holds

$$
\left\|\mathrm{T}_{\mathcal{S}} \sigma \cdot: \mathrm{L}^{\mathrm{p}}(\sigma) \rightarrow \mathrm{L}^{\mathrm{q}}(w)\right\| \lesssim[w, \sigma]_{\mathfrak{p}, \varepsilon_{\mathfrak{p}}}^{\frac{1}{\mathrm{p}}}+[\sigma, w]_{\mathfrak{p}^{\prime} \varepsilon_{\mathfrak{p}^{\prime}}}^{\frac{1}{p^{\prime}}} \cdot
$$

2010 Mathematics Subject Classification. 42B20, 42B25.

Key words and phrases. Sparse Operator, Weighted Inequalities, Entropy Bumps, Calderón-Zygmund Operator. 
For our next theorem, let

$$
[[w, \sigma]]_{p, \alpha_{p}}:=\sup _{Q}\langle w\rangle_{Q}\langle\sigma\rangle_{Q}^{p-1} \alpha_{p}\left(\langle\sigma\rangle_{Q}\right)
$$

where $\alpha_{p}$ is a function that is decreasing on $(0,1)$ and increasing on $(1, \infty)$ and that satisfies $\sum_{r \in \mathbb{Z}} \alpha_{p}\left(2^{-r}\right)^{-\frac{1}{p}}<\infty$. We have

Theorem 1.2. With definitions and above, and $1<p<\infty$ there holds

$$
\left\|T_{\mathcal{S}} \sigma \cdot: L^{p}(\sigma) \rightarrow L^{p}(w)\right\| \lesssim[[w, \sigma]]_{p}^{\frac{1}{p}, \alpha_{\mathfrak{p}}}+[[\sigma, w]]_{\mathfrak{p}^{\prime}, \alpha_{\mathfrak{p}}}^{\frac{1}{p^{\prime}}} \cdot
$$

The type of theorems we are proving are known as "bumps", because they slightly strengthen the joint $A_{p}$ characteristic. The bumps in Theorem 1.1 were introduced in [19] and are known as "entropy bumps", and the bumps in Theorem 1.2 seem to be new. There is a long history of theorems of this type (see for example $[2-5,7,8,10,14-16]$ ) but in [19] it is shown that under some mild conditions, the entropy bumps are smaller than other bumps and so this approach is more robust.

Our proof builds on the ideas in [13] and uses an interesting formula by Hytönen in [6] that generalizes the expansion of sums like $\left(\sum_{j} a_{j}\right)^{2}$ to powers other than 2 . This formula seems to be powerful and it seems to have been first observed in [6].

\section{The ProOF}

The proof will use the following facts and notation. First, all cubes considered below are in the sparse collection $\mathcal{S}$. For a collection $\mathcal{Q}$ of cubes let $[w, \sigma]_{\mathrm{p}}^{\mathcal{Q}}:=\sup _{\mathrm{Q} \in \mathcal{Q}}\langle w\rangle_{\mathrm{Q}}\langle\sigma\rangle_{\mathrm{Q}}^{\mathrm{p}-1}$. The first fact we use is the following deep theorem, originally due to Sawyer. See $[6,11,18]$.

Lemma 2.1. Let $\mathcal{D}$ be a dyadic grid and let $\mathcal{S} \subset \mathcal{D}$ be sparse. Define:

$$
\begin{aligned}
& \mathcal{T}_{1}:=\sup _{\mathrm{P} \in \mathcal{S}} \frac{1}{\sigma(\mathrm{P})} \int_{\mathrm{P}}\left|\sum_{\mathrm{Q} \in \mathcal{S}: \mathrm{Q} \subset \mathrm{P}}\langle\sigma\rangle_{\mathrm{Q}} \pi_{\mathrm{Q}}(x)\right|^{\mathrm{p}} w(x) \mathrm{d} x, \\
& \mathcal{T}_{2}:=\sup _{\mathrm{P} \in \mathcal{S}} \frac{1}{\mathcal{w}(\mathrm{P})} \int_{\mathrm{P}}\left|\sum_{\mathrm{Q} \in \mathcal{S}: \mathrm{Q} \subset \mathrm{P}}\langle w\rangle_{\mathrm{Q}} \pi_{\mathrm{Q}}(x)\right|^{\mathrm{p}^{\prime}} \sigma(x) \mathrm{d} x .
\end{aligned}
$$

Then:

$$
\left\|\mathrm{T}_{\mathcal{S}} \sigma \cdot: \mathrm{L}^{\mathrm{p}}(\sigma) \rightarrow \mathrm{L}^{\mathrm{p}}(w)\right\| \lesssim \mathcal{T}_{1}^{\frac{1}{\mathrm{p}}}+\mathcal{T}_{2}^{\frac{1}{\mathrm{p}^{\prime}}}
$$

We first give the proof of Theorem 1.1 in the case $p=2$.

Proof of Theorem 1.1 when $p=2$. We will verify the testing conditions hold; we will only verify the first condition as the second condition is verified similarly. Fix $\mathrm{P} \in \mathcal{S}$. By the 
triangle inequality and the summability condition of $\varepsilon_{2}$, it suffices to show

$$
\int_{\mathrm{P}}\left|\sum_{\mathrm{Q} \in \mathcal{Q}_{\mathrm{r}}}\langle\sigma\rangle_{\mathrm{Q}} \pi_{\mathrm{Q}}\right|^{2} w \lesssim \frac{1}{\varepsilon_{2}\left(2^{\mathrm{r}}\right)}[\sigma, w]_{2, \varepsilon_{2}} \sigma(\mathrm{P}),
$$

where $\mathcal{Q}_{\mathrm{r}}:=\left\{\mathrm{Q}: \mathrm{Q} \subset \mathrm{P}\right.$ and $\left.\rho_{\sigma}(\mathrm{Q}) \simeq 2^{r}\right\}$ for $r \in \mathbb{N}$. Since two cubes in $\mathcal{Q}_{\mathrm{r}}$ are either nested or disjoint, there holds

$$
\left|\sum_{\mathrm{Q} \in \mathcal{Q}_{\mathrm{r}}}\langle\sigma\rangle_{\mathrm{Q}} \pi_{\mathrm{Q}}(x)\right|^{2} \simeq \sum_{\mathrm{Q} \in \mathcal{Q}_{\mathrm{r}}} \sum_{\mathrm{Q}^{\prime} \subset \mathrm{Q}}\langle\sigma\rangle_{\mathrm{Q}}\langle\sigma\rangle_{\mathrm{Q}^{\prime}} \boldsymbol{1}_{\mathrm{Q}^{\prime}}(x) .
$$

Inserting this into (2.1), and using $\rho_{\sigma}(\mathrm{Q}) \simeq 2^{r}$ for $\mathrm{Q} \in \mathcal{Q}_{\mathrm{r}}$,

$$
\begin{aligned}
\int_{\mathrm{P}}\left|\sum_{\mathrm{Q} \in \mathcal{Q}_{r}}\langle\sigma\rangle_{\mathrm{Q}} \pi_{\mathrm{Q}}\right|^{2} w & \simeq \sum_{\mathrm{Q} \in \mathcal{Q}_{r}} \sum_{\mathrm{Q}^{\prime} \subset \mathrm{Q}}\langle\sigma\rangle_{\mathrm{Q}}\langle\sigma\rangle_{\mathrm{Q}^{\prime}} w\left(\mathrm{Q}^{\prime}\right) \\
& =\sum_{\mathrm{Q} \in \mathcal{Q}_{\mathrm{r}}}\langle\sigma\rangle_{\mathrm{Q}} \sum_{\mathrm{Q}^{\prime} \subset \mathrm{Q}}\left|\mathrm{Q}^{\prime}\right|\langle\sigma\rangle_{\mathrm{Q}^{\prime}}\langle w\rangle_{\mathrm{Q}^{\prime}} \frac{\rho_{\sigma}(\mathrm{Q}) \varepsilon\left(\rho_{\sigma}(\mathrm{Q})\right)}{\rho_{\sigma}(\mathrm{Q}) \varepsilon\left(\rho_{\sigma}(\mathrm{Q})\right)} . \\
& \lesssim \frac{1}{2^{r} \varepsilon_{2}\left(2^{r}\right)}[\sigma, w]_{2, \varepsilon_{2}} \sum_{\mathrm{Q} \in \mathcal{Q}_{r}}\langle\sigma\rangle_{\mathrm{Q}} \sum_{\mathrm{Q}^{\prime} \subset \mathrm{Q}}\left|\mathrm{Q}^{\prime}\right| .
\end{aligned}
$$

Since $\mathcal{Q}_{\mathrm{r}}$ is sparse, $\sum_{\mathrm{Q} \in \mathcal{Q}_{\mathrm{r}}}\langle\sigma\rangle_{\mathrm{Q}} \sum_{\mathrm{Q}^{\prime} \subset \mathrm{Q}}\left|\mathrm{Q}^{\prime}\right| \lesssim \sum_{\mathrm{Q} \in \mathcal{Q}_{\mathrm{r}}} \sigma(\mathrm{Q})$.

Set $\mathcal{Q}_{r}^{*}$ to be the maximal cubes in $\mathcal{Q}_{r}$. Using the fact that $\left|\mathrm{E}_{\mathrm{Q}}\right| \simeq|\mathrm{Q}|$ and that $\left\{\mathrm{E}_{\mathrm{Q}}\right\}$ are pairwise disjoint, there holds:

$$
\text { (2.2) } \sum_{\mathrm{Q} \in \mathcal{Q}_{\mathrm{r}}} \sigma(\mathrm{Q}) \simeq \sum_{\mathrm{Q}^{*} \in \mathcal{Q}_{\mathrm{r}}^{*}} \int_{\mathrm{Q}^{*}} \sum_{\mathrm{Q} \subset \mathrm{Q}^{*}}\langle\sigma\rangle_{\mathrm{Q}} \mathbb{1}_{\mathrm{E}_{\mathrm{Q}}} \leq \sum_{\mathrm{Q}^{*} \in \mathcal{Q}_{\mathrm{r}}^{*}} \int_{\mathrm{Q}^{*}} \mathrm{M}\left(\sigma \mathbb{1}_{\mathrm{Q}^{*}}\right) \leq 2^{\mathrm{r}} \sum_{\mathrm{Q}^{*} \in \mathcal{Q}_{\mathrm{r}}^{*}} \sigma\left(\mathrm{Q}^{*}\right) \text {. }
$$

Since the cubes in $\mathcal{Q}_{r}^{*}$ are pairwise disjoint, the sum is bounded by $\sigma(\mathrm{P})$, as desired.

To use a similar idea for $p \neq 2$ we need the following lemma proven in [6].

Lemma 2.2. Let $\mathcal{Q}$ be any collection of cubes. With obvious notation there holds

$$
\int_{\mathrm{P}}\left(\sum_{\mathrm{Q} \in \mathcal{Q}: \mathrm{Q} \subset \mathrm{P}}\langle\sigma\rangle_{\mathrm{Q}} \boldsymbol{1}_{\mathrm{Q}}\right)^{\mathrm{p}} w \lesssim[w, \sigma]_{\mathrm{p}}^{\mathcal{Q}} \sum_{\mathrm{Q} \subset \mathrm{P}}\langle\sigma\rangle_{\mathrm{Q}}|\mathrm{Q}| \text {. }
$$

We use this to prove Theorem 1.1 for all $p>1$.

Proof of Theorem 1.1. We will verify the testing conditions. For simplicity, we will verify the first condition; the dual condition is verified similarly. Thus, let $\mathrm{P}$ be any cube in $\mathcal{D}$. For $r \geq 0$, let $\mathcal{Q}_{r}=\left\{Q \subset P: \rho_{\sigma}(Q) \simeq 2^{r}\right\}$. Note that for these cubes, there holds

$$
[w, \sigma]_{\mathfrak{p}}^{\mathcal{Q}} \lesssim \frac{1}{2^{r} \varepsilon_{\mathfrak{p}}\left(2^{r}\right)}[w, \sigma]_{p, \varepsilon_{p}} .
$$


Therefore, by the triangle inequality and Lemma 2.2 there holds

$$
\begin{aligned}
\left(\int_{\mathrm{P}}\left(\sum_{\mathrm{Q}: \mathrm{Q} \subset \mathrm{P}}\langle\sigma\rangle_{\mathrm{Q}} \pi_{\mathrm{Q}}\right)^{\mathrm{p}} w\right)^{\frac{1}{\mathrm{p}}} & \leq \sum_{\mathrm{r} \geq 0}\left(\int_{\mathrm{P}}\left(\sum_{\mathrm{Q} \in \mathcal{Q}_{r}}\langle\sigma\rangle_{\mathrm{Q}} \pi_{\mathrm{Q}}\right)^{\mathrm{p}} w\right)^{\frac{1}{\mathrm{p}}} \\
& \lesssim[w, \sigma]_{\mathfrak{p}, \varepsilon_{\mathfrak{p}}}^{\frac{1}{p}} \sum_{\mathrm{r} \geq 0} \frac{1}{\varepsilon_{\mathfrak{p}}\left(2^{r}\right)^{\frac{1}{p}}}\left(\frac{1}{2^{r}} \sum_{\mathrm{Q} \in \mathcal{Q}_{r}} \sigma(\mathrm{Q})\right)^{\frac{1}{p}} \\
& \lesssim[w, \sigma]_{p}^{\frac{1}{p}, \varepsilon_{\mathfrak{p}}} \sum_{\mathrm{r} \geq 0} \frac{1}{\varepsilon_{\mathfrak{p}}\left(2^{r}\right)^{\frac{1}{p}}} \sigma(\mathrm{P})^{\frac{1}{p}}
\end{aligned}
$$

In the last estimate, we used the fact that for the cubes in $\mathcal{Q}_{r}, \rho_{\sigma}(\mathrm{Q}) \simeq 2^{\mathrm{r}}$ and so we can use the same estimate as in (2.2). The summability condition on $\varepsilon_{\mathrm{p}}$ completes the proof.

We conclude with the proof of Theorem 1.2.

Proof of Theorem 1.2. As above we need to verify the testing conditions, and we will only verify the first. Thus, let $\mathrm{P}$ be any cube in $\mathcal{D}$. For $r \in \mathbb{Z}$ let $\mathcal{Q}_{r}=\left\{\mathrm{Q} \subset \mathrm{P}:\langle\sigma\rangle_{\mathrm{Q}} \simeq 2^{r}\right\}$. Using the summability condition on $\alpha_{p}$, as in the proof of Theorem 1.1 we may assume that all cubes are contained in $\mathcal{Q}_{r}$.

Again let $\mathcal{Q}_{\mathrm{r}}^{*}$ denote the maximal cubes in $\mathcal{Q}_{\mathrm{r}}$. Using Lemma 2.2, there holds

$$
\begin{aligned}
\int_{\mathrm{P}}\left(\sum_{\mathrm{Q} \in \mathcal{Q}_{r}}\langle\sigma\rangle_{\mathrm{Q}} \pi_{\mathrm{Q}}\right)^{\mathrm{p}} w & \lesssim \frac{1}{\alpha_{\mathrm{p}}\left(2^{\mathrm{r}}\right)}[[w, \sigma]]_{\mathrm{p}, \alpha_{\mathrm{p}}} \sum_{\mathrm{Q} \in \mathcal{Q}_{r}}\langle\sigma\rangle_{\mathrm{Q}}|\mathrm{Q}| \\
& \simeq \frac{1}{\alpha_{\mathrm{p}}\left(2^{\mathrm{r}}\right)}[[w, \sigma]]_{\mathrm{p}, \alpha_{\mathrm{p}}} \sum_{\mathrm{Q}^{*} \in \mathcal{Q}_{\mathrm{r}}^{*}} \sum_{\mathrm{Q} \subset \mathrm{Q}^{*}}|\mathrm{Q}| \\
& \simeq \frac{1}{\alpha_{\mathrm{p}}\left(2^{\mathrm{r}}\right)}[[w, \sigma]]_{\mathrm{p}, \alpha_{\mathrm{p}}} \sum_{\mathrm{Q}^{*} \in \mathcal{Q}_{r}^{*}}|\mathrm{Q}|^{*} .
\end{aligned}
$$

In the second line we used the definition of $\mathcal{Q}_{r}$ and in the third line we used sparseness. Again, using the definition of $\mathcal{Q}_{\mathrm{r}}$, the sum is equivalent to $\sum_{\mathrm{Q}^{*} \in \mathcal{Q}_{\mathrm{r}}^{*}} \sigma\left(\mathrm{Q}^{*}\right)$ and by the maximality of the cubes in $\mathcal{Q}^{*}$, it follows that this sum is dominated by $\sigma(P)$.

Acknowledgment.R. Rahm would like to thank Kabe Moen for telling him about the formula from [6].

\section{REFERENCES}

[1] José M. Conde-Alonso and Guillermo Rey, A pointwise estimate for positive dyadic shifts and some applications, Math. Ann. 365 (2016), no. 3-4, 1111-1135.

[2] D. Cruz-Uribe, J. M. Martell, and C. Pérez, Sharp two-weight inequalities for singular integrals, with applications to the Hilbert transform and the Sarason conjecture, Adv. Math. 216 (2007), no. 2, 647-676, DOI 10.1016/j.aim.2007.05.022. MR2351373 
[3] David V. Cruz-Uribe, José Maria Martell, and Carlos Pérez, Weights, extrapolation and the theory of Rubio de Francia, Operator Theory: Advances and Applications, vol. 215, Birkhäuser/Springer Basel AG, Basel, 2011. MR2797562

[4] David Cruz-Uribe and Kabe Moen, One and two weight norm inequalities for Riesz potentials, Illinois J. Math. 57 (2013), no. 1, 295-323.

[5] David Cruz-Uribe, Alexander Reznikov, and Alexander Volberg, Logarithmic bump conditions and the two-weight boundedness of Calderón-Zygmund operators, Adv. Math. 255 (2014), 706-729, DOI 10.1016/j.aim.2014.01.016. MR3167497

[6] Tuomas P. Hytönen, The $A_{2}$ theorem: remarks and complements, Harmonic analysis and partial differential equations, Contemp. Math., vol. 612, Amer. Math. Soc., Providence, RI, 2014, pp. 91106.

[7] Tuomas P. Hytönen and Michael T. Lacey, The $A_{p}-A_{\infty}$ inequality for general Calderón-Zygmund operators, Indiana Univ. Math. J. 61 (2012), no. 6, 2041-2092, DOI 10.1512/iumj.2012.61.4777. MR3129101

[8] Michael T. Lacey, On the Separated Bumps Conjecture for Calderón-Zygmund Operators, Hokkaido Math. J. 45 (2016), no. 2, 223-242, DOI 10.14492/hokmj/1470139402. MR3532130

[9] Andrei K. Lerner, A pointwise estimate for the local sharp maximal function with applications to singular integrals, Bull. Lond. Math. Soc. 42 (2010), no. 5, 843-856.

[10] Andrei K. Lerner and Kabe Moen, Mixed $A_{p}-A_{\infty}$ estimates with one supremum, Studia Math. 219 (2013), no. 3, 247-267.

[11] Michael T. Lacey, Eric T. Sawyer, and Ignacio Uriarte-Tuero, Two Weight Inequalities for Discrete Positive Operators (2009), available at http://arxiv.org/abs/0911.3437.

[12] Michael T. Lacey, An Elementary Proof of the $A_{2}$ Bound (2015), available at http://arxiv.org/abs/1501.05818.

[13] Michael T. Lacey and Scott Spencer, On entropy bumps for Calderón-Zygmund operators, Concr. Oper. 2 (2015), 47-52.

[14] Fedor Nazarov, Alexander Reznikov, Sergei Treil, and Alexander Volberg, A Bellman function proof of the $\mathrm{L}^{2}$ bump conjecture, J. Anal. Math. 121 (2013), 255-277.

[15] C. J. Neugebauer, Inserting $A_{p}$-weights, Proc. Amer. Math. Soc. 87 (1983), no. 4, 644-648.

[16] C. Pérez, Weighted norm inequalities for singular integral operators, J. London Math. Soc. (2) 49 (1994), no. 2, 296-308.

[17] Carlos Pérez, On sufficient conditions for the boundedness of the Hardy-Littlewood maximal operator between weighted $\mathrm{L}^{\mathrm{p}}$-spaces with different weights, Proc. London Math. Soc. (3) 71 (1995), no. 1, 135-157.

[18] Eric T. Sawyer, A characterization of two weight norm inequalities for fractional and Poisson integrals, Trans. Amer. Math. Soc. 308 (1988), no. 2, 533-545.

[19] Sergei Treil and Alexander Volberg, Entropy conditions in two weight inequalities for singular integral operators, Adv. Math. 301 (2016), 499-548.

Robert Rahm, School of Mathematics, Washington University in St. Louis, One Brookings Drive, St. Louis, MO USA 63112

E-mail address: robertrahm@gmail.com

Scott Spencer, School of Mathematics, Georgia Institute of Technology, 686 Cherry Street, Atlanta, GA USA 30332-0160

E-mail address: spencer@math.gatech.edu 